\title{
EL SEMIGRUPO DE VALORES DE UNA CURVA PLANA IRREDUCTIBLE ALGEBROIDE
}

\author{
Frank Collantes ${ }^{1}$, Marlo $_{\text {Carranza }}{ }^{2}$ \\ (Recibido: 07/10/2015 - Aceptado: 26/10/2015)
}

Resumen: Dada una curva, tenemos en virtud al teorema de Newtón-Puiseux que ella posee solución en la cerradura algebraica de

$$
\mathbb{C}((X))^{*}=\bigcup_{n=1}^{\infty} \mathbb{C}\left(\left(X^{\frac{1}{n}}\right)\right) .
$$

Sea $\varphi\left(X^{\frac{1}{n}}\right)=\sum_{i \geq 1} b_{i} X^{\frac{1}{n}} \in \mathbb{C}((X))^{*}$ tal que $f\left(X, \varphi\left(X^{\frac{1}{n}}\right)\right)=0$. Esto nos lleva a representar a $f$ en forma paramétrizada, $X=T^{n}, Y=\sum_{i \geq 1} b_{i} T^{i}$.

Mostramos resultados vitales que servirán posteriormente para desarrollar investigación sobre la teoría de singularidades de curvas algebroides planas ya sea esta representada en forma de Weierstrass o en su forma parametrizada.

Palabras clave: Curvas algebroides planas, transformada cuadrática, (a)-equivalencia, contacto maximal, género de una curva.

\section{THE SEMIGROUP OF VALUES OF AN IRREDUCIBLE PLANE CURVE ALGEBROID}

\begin{abstract}
In this paper we present important results on the theory of irreducible plane curves, vital to develop research on the theory of singularities of plane curves algebroids is represented either in the form of Weierstrass or formal power series.
\end{abstract}

Keywords: Algebroids planar curves, quadratic transformation, (a)-equivalence, maximal contact, a genus curve.

\section{Introducción}

Los estudios de semigrupo de una curva plana irreducible algebroide plana sobre un cuerpo algebraicamente fechado $\mathrm{K}$ son hechos principalmente en el caso $\operatorname{char}(K)=0$. La razón principal es que en ese caso pueden ser usados las series de Puiseux. En este trabajo demostraremos, (sin uso de tales métodos o resultados, conocidos en el caso de $\operatorname{char}(K)=0$,) sobre la estructura de los semigrupos de curvas planas irreducibles algebroides sobre cuerpos algebraicamente cerrados de característica cualquiera. Además de eso damos una caracterización de los semigrupos, que ocurren como semigrupo de curvas planas sobre un cuerpo algebraicaimente cerrado $K$, una caracterización que hasta parece nueva si $\operatorname{char}(K)=0$. Nuestro método son las llamadas las sucesiones de Apery, que primeramente fueron usadas por Apery y después estudiados por Acebedo con eso determinamos el menor sistema de generadores de un semigrupo de una curva plana irreducible algebroide y además de eso mostraremos que ese menor sistema de generadores

\footnotetext{
${ }^{1}$ UNMSM, Facultad de Ciencias Matemáticas, e-mail: E-mail: frank1400113@hotmail.com

${ }^{2}$ UNMSM, Facultad de Ciencias Matemáticas, e-mail: E-mail: marlocpx5@hotmail.com
} 
y los pares característicos en sentido de Hoch se determinan mutuamente, eso generaliza los teoremas de Abihyinkar y Hoch y Acebedo. También una caracterización de los semigrupos, que son semigrupos de curvas planas irreducibles algebroides es hecha con esas sucesiones, además de eso con ayuda de las secuencias de Apery obtenemos aplicaciones sobre ecuaciones lineales diofanticas, obtenemos un teorema sobre la conocida desigualdad sobre el conductor de un semigrupo, que contiene un teorema de Brawer y Sulvinder y que refina un teorema de Berlin y Carbone, además de eso computamos con esas sucesiones el conductor de progresiones.

El trabajo consiste en dos partes, la primera tratamos los semigrupo y la aplicación sobre ecuaciones lineales diofanticas. En la segunda estudiaremos los semigrupos de curvas planas irreducible algebroides.

\section{Semigrupo de los Números Naturales}

En este capitulo definimos los semigrupos y también de sus conjuntos generadores, luego definimos el conductor que un invariante que caracteriza a un semigrupo. Después definimos las sucesiones de Apery y escribimos algunas de sus propiedades, así como también definimos las sucesiones agradables, para luego estudiar las sucesiones de Apery fuertemente crecientes.

\subsection{Semigrupos de los naturales}

Un semigrupo, $H$ es un subconjunto de los naturales, conteniendo al cero y cerrado para la adición. Consideremos que nuestro semigrupo $H$ tenga la propiedad de $\operatorname{mcd}(H)=1$

Definición 2.1 $H$ contiene casi todos los números naturales, el menor número $n$ tal que $[n, \infty[\subset H$ es llamado el conductor de $H$.

Definimos

$$
e_{0}=\operatorname{mín}\{H \backslash\{0\}\}, \ldots, e_{i+1}=\operatorname{mín}\left\{H \backslash \sum_{k=0}^{i} e_{k} \mathbb{N}\right\}, i=1, \ldots, s-1 \text {, }
$$

se tiene que $e_{0}<e_{1}<\ldots<e_{s}$.

$s \leq e_{0}$. En efecto, para $e_{i}$ y $e_{0}$, Por el algoritmo de la division existe $k$ y $j$ tal que $e_{i}=e_{0} k+j$ con $0 \leq j<e_{0}$, esto es $e_{i} \in[j]_{e_{0}}$. Si $s=0$, si, solamente si, $H=\mathbb{N}$.

De la definición podemos ver que $H$ es finitamente generado por $E=\left\{e_{0}, \ldots, e_{s}\right\}$.

\section{2. $\quad$ Sucesiones de Apery}

Sea $0 \neq p \in H$. Como $H$ contiene casi todos los números naturales, $H$ tiene intersección no vacía con cada clase módulo $p$ y el conjunto

$$
A_{p}=\{\operatorname{mín}\{H \cap n+p \mathbb{Z}\} / n \in \mathbb{N}\}
$$

tiene $p$ elementos y generan $A_{p}=\left\{a_{0}, \ldots, a_{p-1}\right\}$ con $0=a_{0}<a_{1}<\cdots<a_{p-1}$.

Definición 2.2 $A_{p}$ es llamado el conjunto de Apery de $H$ con respecto a $p$, si

$$
p=\min \{H \backslash\{0\}\},
$$

diremos que $A_{p}$ es un Apery conjunto de $H$.

A los $a_{0}, \ldots, a_{p-1}$ es llamado el Apery sucesión de $H$ con respecto a $p$, si $p=\operatorname{mín}\{H \backslash\{0\}\}$, diremos simplemente que $A_{p}$ es Apery sucesión de $H$. 
Cada número $n \in \mathbb{Z}$ por el algoritmo de la división se escribe de manera única en la forma

$$
n=a_{i}+m p \text { con } 0 \leq i \leq p-1, m \in \mathbb{Z} .
$$

Sigue que para $p \in H$

$$
n \in H \text { si, solo si, } m \geq 0 \text {. }
$$

En efecto si $m<0$ sigue $-m>0$ luego $n<n+(-m) p$, lo que es una contradiición pues $n \in H \quad$ y la definición de $a_{i}=n+(-m) p$. Por tanto $m \geq 0$.

Por definición de la Apery sucesión tenemos que

$$
n \in A_{p} \text { si, solo si, } n \in H, \quad n-p \notin H \text {. }
$$

Consecuentemente

$$
H=\bigcup_{i=0}^{p-1} a_{i}+p \mathbb{N}
$$

y por tanto $p, a_{1}, \ldots, a_{p-1}$ es un sistema de generadores de $H$.

Si en particular $1<p=\operatorname{mín}\{H \backslash\{0\}\}$ y $E=\left\{e_{0}, \ldots, e_{s}\right\}$ es el menor sistema de generadores de $H$, entonces

$$
E \subseteq\left\{p, a_{1}, \ldots, a_{p-1}\right\}, e_{0}=p, e_{1}=a_{1}
$$

Lema 2.3 Sea $H$ un semigrupo, y $a_{0}, \ldots, a_{p-1}$ la Apery sucesión. Entonces, son equivalentes.

1. $H$ es generado por dos elementos (i.e $s=1$ )

2. $a_{i}=i a_{1} \quad$ para $i=0, \ldots, p-1$

3. $H=\bigcup_{i=0}^{p-1} i a_{1}+p \mathbb{N}$

Demostración 1) $\Rightarrow 2)$ Sea $H=\left\langle p, a_{1}\right\rangle$, sigue $a_{i}=\alpha p+\beta a_{1}$ con $\alpha, \beta \geq 0$, luego $\beta a_{1}=a_{i}-\alpha p$ con $-\alpha \geq 0$, por tanto $\alpha=0$, consecuentemente

$$
a_{i}=\beta_{i} a_{1} .
$$

Sea $a_{2}-a_{1}=a_{j}+m p$, con $m \geq 0$, sigue $a_{j}+a_{1}=a_{2}+m p \in H$, luego $m \leq 0$, por tanto $m=0$. Consecuentemente $a_{2}=a_{1}+a_{j}$, y $j<2$.

$j \neq 0$ si no $j=0$ sigue $a_{2}=a_{1}+p$, entonces $a_{2}-p \in H$, que es una contradicción. Consecuentemente $j=1$ y luego $a_{2}=2 a_{1}$

2) $\Rightarrow 3$ ) Es evidente de 4

$3) \Rightarrow 1$ ) Sea $m \in H$, luego $m \in i_{0} a_{1}+p \mathbb{N}$ sigue $m=i_{0} a_{1}+p k$, para algún $k \in \mathbb{N}$, consecuentemente $H$ es generado por $a_{1}, p$.

Ejemplo 2.4 Sea

$$
H=\{0,4,7,8,11,14,15,16,18,19,20,21,22, \ldots\}
$$

En este caso $s \leq e_{0}=\operatorname{mín}\{H \backslash\{0\}\}=4$, luego $e_{1}=\operatorname{mín}\{H \backslash 4 \mathbb{N}\}=7, e_{2}=\operatorname{mín}\{H \backslash 4 \mathbb{N}+7 \mathbb{N}\}=$ mín $\emptyset$. Por tanto $H$ es generado por $\{4,7\}$. El conductor de $H$ es $c=18$.

también tenemos la Apery sucesión

$$
A_{p}=\{\text { mín } H \cup n+p \mathbb{Z}\}=\{4,7,14,21\},
$$

observe que $a_{0}=4, a_{1}=7, a_{2}=2 a_{1}, a_{3}=3 a_{1}$.

Lema 2.5 Sea $0 \neq p \in H$ y sea $a_{0}, \ldots, a_{p-1}$ la Apery-sucesión de $H$ a respecto de $P$. Entonces $a_{p-1}-p+1$ es el conductor de $H$ 
Demostración Sea $n \in \mathbb{N}, n \geq a_{p-1}-p+1$, si se escribe de manera única de la forma $n=a_{i}+m p$ con $0 \leq i \leq p-1$, y $m \in \mathbb{Z}$. Si tome $m<0$, entonces $n \leq a_{i}-p \leq a_{p-1}-p<n$ luego $m \geq 0$ i.e. $n \in H$. Como $a_{p-1}-p \notin H$ sigue inmediatamente la afirmación.

Si en particular $p=$ mín $H \backslash\{0\}$ y si $H$ es generado por dos elementos, entonces

$$
(p-1)\left(a_{1}-1\right)=\left(e_{0}-1\right)\left(e_{1}-1\right)
$$

es el conductor de $H$.

Definición 2.6 Un semigrupo $H$ de $\mathbb{N}$ es llamado simétrico, si existe un número $z \in \mathbb{Z}$, tal que para cada entero $n$ vale

$$
n \in H \Leftrightarrow z-n \notin H .
$$

$\mathbb{N} \backslash H$ es el conjunto de las lagunas de $H$.

Proposición 2.7 Si $f$ es el conductor de H, entonces las siguientes afirmaciones son equivalentes.

1. H es simétrico

2. Para cada $n \in \mathbb{Z}$ vale $n \in H \Leftrightarrow f-1-n \notin H$.

3. $|\mathbb{N} \backslash H|=|H \cap\{0,1,2, \ldots, f-1\}|$

4. $2|H \cap\{0,1,2, \ldots, f-1\}|=f$

5. $2|\mathbb{N} \backslash H|=f$

Demostración 1) $\Rightarrow$ 2) Bastará probar que $z=f-1$ en la definición de semigrupo simétrico. En efecto. $z \notin H$ pues $0 \in H$, luego para $m>z$, sigue $z-m<0$ luego $z-m \notin H$, entonces $m \in H$, consecuentemente $z=f-1$.

2) $\Rightarrow 3)$ Sea

$$
\begin{array}{ccc}
\Phi: H \cap\{0,1,2, \ldots, f-1\} & \longrightarrow & \mathbb{N} \backslash H \\
n & \rightarrow \Phi(n)=f-1-n
\end{array}
$$

$\Phi$ es biyección. En efecto, sean $n_{1}, n_{2} \in H \cap\{0,1,2, \ldots, f-1\}$ con $n_{1} \neq n_{2}$, sigue $f-1-n_{1} \neq f-1-n_{2}$, luego $\Phi\left(n_{1}\right) \neq \Phi\left(n_{2}\right)$, por tanto $\Phi$ es inyectivo. Sea $z \in \mathbb{N} \backslash H$, sigue $z \notin H$, luego existe $n_{1}=f-1-z \in H$, con $0 \leq n_{1} \leq f-1$, por tanto $\Phi$ es sobreyectivo. Consecuentemente

$$
|\mathbb{N} \backslash H|=|H \cap\{0,1,2, \ldots, f-1\}|
$$

3) $\Rightarrow 1$ ) Sea $z=f-1 \in \mathbb{Z}$.

Si $n \in H$, entonces $z-n=f-1-n \notin H$. Si no es así $z-n \in H$ y $f-1=n+(z-n) \in H$, que es un absurdo.

Si $z-n \notin H$, sigue $f-1-n \notin H$, luego $n \in H$

$i i i), i v)$ y $v$ ) son evidentemente equivalentes.

Proposición 2.8 Sea $0 \neq p \in H$, y sea $a_{0}, a_{1}, \ldots, a_{p-1}$, la Apery-sucesión de $H$ a respecto de $p$. Entonces son equivalentes

1. H es simétrico.

2. Para cada $n \in \mathbb{Z}$ se tiene $n \in H \Leftrightarrow a_{p-1}-p-n \notin H$.

3. Para $i=0,1, \ldots, p-1$ se tiene $a_{p-1}-a_{i} \in\left\{a_{0}, a_{1}, \ldots, a_{p-1}\right\}$.

4. Para cada $i=0,1, \ldots, p-1$ se tiene $a_{p-1}=a_{i}+a_{p-1-i}$. 
Demostración (1) $\Rightarrow(2)$ Para $n \in \mathbb{Z} \quad$ y $f=a_{p-1}-p+n$ el conductor de $H$. Sigue de la proposición 2.7 que

$$
n \in H \Leftrightarrow a_{p-1}-p-n \notin H
$$

(2) $\Rightarrow(3)$ Para $0 \leq i \leq p-1, \quad a_{p-1}-p-a_{i} \in \mathbb{Z}$, sigue $a_{p-1}-p-a_{i} \notin H$, luego por (2) $a_{p-1}-p-a_{i}=a_{j}+m p$ con $m<0$ y $0 \leq j \leq p-1$.

Si $m<-1$, sigue

$$
a_{p-1}-p-\left(a_{i}-p\right)=a_{j}+(m+1) p \notin H,
$$

luego $a_{i}-p \in H$. Que es una contradicción con 3. Por tanto $m=-1$ y $a_{p-1}-a_{i}=a_{j}$.

$(3) \Rightarrow(4)$ Se tiene que $a_{p-1}-a_{i}=a_{j_{i}}$. Además por la definición de Apery-sucesión tenemos que $a_{0}<a_{1}<\ldots<a_{p-1}$. Sigue

$$
a_{j_{0}}>a_{j_{1}}>\ldots>a_{j_{i}} \ldots>a_{j_{p-1}}
$$

consecuentemente $a_{i}+a_{p-i+1}=a_{p-1}$

$(4) \Rightarrow(1)$ Sea $z=f-1=a_{p-1}-p$. Luego, si $n \in H$, sigue $n=a_{j}+m p$ con $m \geq 0$. Luego $z-n=a_{p-1}-p-a_{j}-m p=a_{p-j-1}+(-m) p \notin H$. Recíprocamente si $z-n \notin H$, sigue $z-n=a_{j}+m p$, con $m<0$, luego $n=a_{p-1}-p-a_{j}-m p=a_{p-j-1}-(m+1) p$ com $-(m+1) \geq 0$, consecuentemente $n \in H$.

En particular, si $H$ es generado por dos elementos, entonces la observación 2.8 muestra que $H$ es simétrico. Como los ejemplos de los subsemigrupos $\{0\} \cup\{n, n+1, \ldots, \infty\}$ de $\mathbb{N}$ para $n \geq 3$ muestran, eso no siempre es verdadero si el número de generadores mínimos es mayor que dos.

\subsection{Sucesiones Agradables}

Definición 2.9 Sea la sucesión de enteros positivos no necesariamente monótona $t_{0}, t_{1}, \ldots, t_{r}$, definimos

1. La sucesión acoplada $g_{0}, g_{1}, \ldots, g_{r}$, donde

$$
g_{0}=t_{0}, \quad g_{i}=\operatorname{mcd}\left(t_{0}, \ldots, t_{i}\right), i=1,2, \ldots, r
$$

2. La sucesión colada $n_{0}, n_{1}, \ldots, n_{r}$, donde

$$
n_{0}=1, \quad n_{i}=\frac{g_{i-1}}{g_{i}}, i=1,2, \ldots, r
$$

3. La sucesión a mano $m_{0}, m_{1}, \ldots, m_{r}$, donde

$$
m_{0}=1, \quad t_{i}=g_{i} m_{i}, i=1,2, \ldots, r
$$

Lema 2.10 Dada la sucesión acoplada $g_{0}, g_{1}, \ldots, g_{r}, y$ la sucesión colada $n_{0}, n_{1}, \ldots, n_{r}$. Entonces

1. $g_{i}=n_{i+1} \ldots n_{r-1} n_{r}, \quad i=1, \ldots, r$.

2. $g_{i} \mid g_{i-1}, \quad i=1, \ldots, r$.

3. $\left(m_{i}, n_{i}\right)=1$.

Demostración Es una simple verificación de la definición. 
Definición 2.11 Dada la sucesión $t_{0}, t_{1}, \ldots, t_{r}$ como en 1,2 se define la sucesión derivada de enteros sin divisores comunes $t_{0}^{\prime}, t_{1}^{\prime}, \ldots, t_{r}^{\prime}$, donde

$$
t_{i}^{\prime} n_{r}=t_{i}, \quad i=0,1, \ldots, r-1
$$

De manera similar se definen los la sucesión acoplada derivada $g_{i}^{\prime}$ para $i=1, \ldots, r-1$. La sucesión colada derivada $n_{i}^{\prime}$ para $i=1 \ldots, r-1$. Y la sucesión a mano derivada $m_{i}^{\prime}$ para $i=1, \ldots, r-1$.

Proposición 2.12 Tenemos que $g_{i}=n_{r} g_{i}^{\prime}, \quad n_{i}=n_{i}^{\prime}$ y $m=m_{i}^{\prime}$ para $i=1 \ldots, r-1$

Demostración La prueba es evidente de la definición.

Proposición 2.13 Cada numero natural $n<t_{0}$ es representado de manera única como

$$
n=\sum_{i=1}^{r} s_{i} n_{1} \ldots n_{i-1} .
$$

Demostración Sea la sucesión colada $n_{1}, n_{2}, \ldots, n_{r}$ y $n<t_{0}$. Entonces para $n$ y $n_{1}$, por el algoritmo de la división, existe $p_{1} \geq 0$ y $s_{1}$, tal que

$$
n=s_{1}+n_{1} p_{1} \text { con } 0 \leq s_{1}<n_{1} .
$$

Nuevamente para $p_{1}$ y $n_{2}$, por el algoritmo de la división existe $p_{2}$ y $s_{2}$ tal que

$$
p_{1}=s_{2}+n_{2} p_{2} \text { con } 0 \leq s_{2}<n_{2}
$$

De 6 y 7 tenemos

$$
n=s_{1}+n_{1} s_{2}+n_{1} n_{2} p_{2} \quad \text { con } 0 \leq s_{2}<n_{2} .
$$

Así sucesivamente llegamos

$$
n=s_{1}+s_{2} n_{1}+\ldots+s_{r-1} n_{1} n_{2} \ldots n_{r-2}+p_{r-1} n_{1} \ldots n_{r-1} \quad \text { con } 0 \leq s_{r-1}<n_{r-1}
$$

Verificaremos que $p_{r-1}<n_{r}$.

En efecto si no fuese así, tenemos que $p_{r-1} \geq n_{r}$, sigue $p_{r-1}=n_{r}+\alpha$ con $\alpha \geq 0$, luego

$$
\begin{aligned}
n= & s_{1}+s_{2} n_{1}+\ldots+n_{1} \ldots n_{r}+n_{1} \ldots n_{r-1} \alpha \\
& =s_{1}+s_{2} n_{1}+\ldots+t_{0}+n_{1} \ldots n_{r-1} \alpha
\end{aligned}
$$

sigue que $n>t_{0}$, lo que es un absurdo. Por tanto $p_{r-1}<n_{r}$, considerando $p_{r-1}=s_{r}$ en (8) el lema esta probado.

Proposición 2.14 Para cada entero $n$ la congruencia $n \cong \sum_{i=1}^{r} s_{i} t_{i}$ modt $_{0}, 0 \leq s_{i}<n_{i}$, $i=1, \ldots, r$ tiene solución única.

Demostración Se probará por inducción sobre $r$.

Para $r=1$, sigue que $g_{1}=1=\left(t_{0}, t_{1}\right)$, luego existe $\alpha$ y $\beta$ enteros tal que

$$
1=t_{0} \alpha+t_{1} \beta
$$

entonces

$$
t_{0} \alpha n+t_{1} \beta n=n
$$

Además por el algoritmo de la division, sigue que para $n \beta$ y $t_{0}$, existe $q$ y $s_{1}$ tal que

$$
n \beta=t_{0} q+s_{1} \text { con } 0 \leq s_{1}<t_{0}=n_{1} .
$$


Entonces de (10) y (9) se tiene que

$$
n=n t_{0} \alpha+t_{1} t_{0} q+t_{1} s_{1}=t_{0}\left(n \alpha+t_{1} q\right)+s_{1} t_{1},
$$

consecuentemente $n \cong s_{1} t_{1} \bmod t_{0}$.

Supongamos válido para $r-1$.

Por el lema 2.10 item 3 se tiene que $\left(m_{r}, n_{r}\right)=1$, luego existe $a$ y $b$ enteros tal que $a m_{r}+b n_{r}=1$, sigue

$$
a n m_{r}+b n n_{r}=n .
$$

Por el algoritmo de la division para an y $n_{r}$, existe $k$ y $s_{r}$ tal que

$$
\text { an }=n_{r} k+s_{r} \text { con } 0 \leq s_{r}<n_{r} .
$$

Reemplazando (12) en (11) sigue

$$
n=s_{r} m_{r}+n^{\prime} n_{r} \text { con } 0 \leq s_{r}<n_{r},
$$

donde $n^{\prime}=k+b n$. Luego para $n^{\prime}$ tenemos por hipótesis inductiva una única representación

$$
n^{\prime} \cong \sum_{i=1}^{r-1} s_{i} t_{i}^{\prime} \bmod t_{0}^{\prime} \quad \text { con } 0 \leq s_{i}<n_{i}^{\prime}=n_{i} .
$$

Multiplicando (14) con $n_{r}$ y reemplazando en (13) sigue

$$
n \cong \sum_{i=1}^{r} s_{i} t_{i} \bmod t_{0}
$$

la unicidad es clara por la construcción.

Corolario 2.15 Supongamos que $H=\sum_{i=0}^{r} \mathbb{N} t_{i}$ es un semigrupo generado por los $t_{i}$ y si $A$ es el Apery-conjunto de $H$ a respecto de $t_{0}$. Entonces para $a \in A$, se tiene una representación única $a=t_{0} s_{0}+\sum_{i=1}^{r} s_{i} t_{i}$, con $0 \geq s_{i}<n_{i}, i=1, \ldots, r$ y $s_{0} \leq 0$.

Demostración Por el lema 2.14 tenemos que para $a=t_{0} s_{0}+\sum_{i=1}^{r} s_{i} t_{i}$, con $0 \geq s_{i}<n_{i}$, $i=1, \ldots, r$. Si $s_{0}>0$ sigue que $a-t_{0} \in H$ que es un absurdo. Por tanto $s_{0} \leq 0$.

Lema 2.16 Cada número natural $n>\sum_{i=1}^{r}\left(n_{i}-1\right) t_{i}-t_{0}$ tiene una única representación

$$
n=\sum_{i=0}^{r} s_{i} t_{i}, s_{i}<n_{i}
$$

para $i=1,2, \ldots, r$ con enteros no negativos $s_{i}$.

Demostración Por el lema 2.14, tenemos una representación única

$$
n=\sum_{i=0}^{r} s_{i} t_{i}
$$

con $0 \leq s_{i}<n_{i}$ para $i=1, \ldots, r$. Como

$$
n>\sum_{i=1}^{r}\left(n_{i}-1\right) t_{i}-t_{0} \geq>\sum_{i=1}^{r} s_{i} t_{i}-t_{0},
$$

sigue $s_{0} t_{0}>-t_{0}$, luego $s_{0} \geq 0$ 
Proposición 2.17 (Teorema de Brauer) Si $H=\sum_{i=0}^{r} \mathbb{N} t_{i}$ es el semigrupo generado por los $t_{i}$. Entonces

$$
f \leq \sum_{i=1}^{r}\left(n_{i}-1\right) t_{i}-t_{0}+1
$$

Demostración Por el lema 2.16 tenemos que para $n \in \mathbb{N}$ se tiene $n \geq \sum_{i=1}^{r}\left(n_{i}-1\right) t_{i}-t_{0}+1$, entonces $n \in H$. Consecuentemente $f \leq \sum_{i=1}^{r}\left(n_{i}-1\right) t_{i}-t_{0}+1$.

Definición 2.18 Sea sucesión $t_{0}, \ldots, t_{r}$, si

$$
n_{i} t_{i} \in \sum_{j<i} \mathbb{N} t_{j}, \quad i=1, \ldots, r
$$

diremos que la sucesión es agradable

Lema 2.19 Sea $t_{0}, t_{1}, \ldots, t_{r}$ una sucesión agradable. Entonces

1. La sucesión derivada es agradable

2. Si eliminamos en una sucesión agradable todos los miembros con $n_{i}=1$, la sucesión permanece agradable y genera el mismo semigrupo.

3. Para $r \leq 1$ cada sucesión es agradable, mas 8,10,11 no es agradable (y también no genera un semigrupo simétrico)

Demostración Sea $t_{0}, t_{1}, \ldots, t_{r}$ una sucesión agradable, sigue existe $\lambda_{1}, \ldots 0, \lambda_{i-1} \in \mathbb{N}$ tal que

$$
n_{i} t_{i}=\lambda_{1} t_{1}+\lambda_{2} t_{2}+\cdots \lambda_{i-1} t_{i-1}
$$

1. Sea $t_{0}^{\prime}, t_{1}^{\prime}, \ldots, t_{r}^{\prime}$ la sucesión derivada. Sigue que $n_{i}^{\prime} t_{i}^{\prime}=n_{i} \frac{t_{i}}{n_{r}}$. De 15 se tiene que

$$
n_{i}^{\prime} t_{i}^{\prime}=\frac{1}{n_{r}}\left(\lambda_{1} t_{1}+\cdots \lambda_{i-1} t_{i-1}\right)=\lambda_{1} t_{1}^{\prime}+\cdots \lambda_{i-1} t_{i-1}^{\prime} .
$$

Por tanto $t_{0}^{\prime}, t_{1}^{\prime}, \ldots, t_{r}^{\prime}$ es agradable.

2. Es suficiente probar que si $n_{k}=1$ para algún $k=0,1, \ldots, r$, entonces la sucesión $t_{0}, \ldots, t_{k-1}, t_{k+1}, \ldots, t_{r}$ es agradable y genera el mismo semigrupo. En efecto, existen $\lambda_{1}, \ldots 0, \lambda_{k-1} \in \mathbb{N}$ tal que

$$
t_{k}=n_{k} t_{k}=\lambda_{1} t_{1}+\lambda_{2} t_{2}+\cdots \lambda_{k-1} t_{k-1}
$$

Para $i<k$ se tiene que

$$
n_{i} t_{i} \in \mathbb{N} t_{1}+\cdots+\mathbb{N} t_{i-1}
$$

Para $i>k$ se tiene que

$$
n_{i} t_{i} \in \mathbb{N} t_{1}+\cdots+\mathbb{N} t_{k-1}+\mathbb{N} t_{k}+\mathbb{N} t_{k+1}+\cdots+\mathbb{N} t_{i-1}
$$

De (16) tenemos que

$$
n_{i} t_{i} \in \mathbb{N} t_{1}+\cdots+\mathbb{N} t_{k-1}+\mathbb{N} t_{k+1}+\cdots+\mathbb{N} t_{i-1}
$$

Por tanto la sucesión $t_{0}, \ldots, t_{k-1}, t_{k+1}, \ldots, t_{r}$ es agradable.

De manera similar se verifica que genera el mismo semigrupo.

Tenemos dos casos:

Primero caso $r=0$ sigue que $t_{0}$ es agradable por definición.

Segundo caso $r=1$, sigue $g_{0}=t_{0}$ y $g_{1}=1$, luego $n_{1}=t_{0}$, por tanto $n_{1} t_{1}=t_{0} t_{1} \in \mathbb{N} t_{0} \mathrm{y}$ $t_{0}, t_{1}$ es agradable. 


\section{Ejemplo 2.20}

1. La sucesión $8,10,11$ no es agradable

2. La sucesión 6,7,8 no es agradable, mas la sucesión $6,8,7$ si es agradable. es agradable.

Teorema 2.21 (Teorema de Angermüller) Sea $t_{0}, t_{1}, \ldots, t_{r}$ una sucesión de los números naturales positivos sin divisor común y sea

$$
v H=\sum_{i=0}^{r} \mathbb{N} t_{i} .
$$

Entonces las siguientes afirmaciones son equivalentes

1. La sucesión $t_{0}, \ldots, t_{r}$ es agradable

2. Cada $n \in H$ posee una representación única $n=\sum_{i=0}^{r} s_{i} t_{i}$ con $0 \leq s_{0}, 0 \leq s_{i}<n_{i}$, $i=1, \ldots, r$

3. El Apery conjunto de $H$ respecto a $t_{0}$ es $A=\left\{\sum_{i=1}^{r} s_{i} t_{i}, 0 \leq s_{i}<n_{i}\right\}$

4. $H$ tiene conductor $f=\sum_{i=1}^{r}\left(n_{i}-1\right) t_{i}-t_{0}+1$

Demostración 1) $\Rightarrow 2$ ) Sea $n \in H$, sigue que existe $x_{0}, x_{1}, \ldots, x_{r} \in \mathbb{N}$, tal que

$$
n=\sum_{i=0}^{r} x_{i} t_{i}, \quad \text { con } x_{i} \geq 0
$$

Luego para $x_{r}$ y $n_{r}$, existe $s_{r}$ y $q_{r}$, tal que

$$
x_{r}=n_{r} q_{r}+s_{r} \text { con } 0 \leq s_{r}<n_{r},
$$

en (17) se tiene

$$
n=x_{0} t_{0}+x_{1} t_{1}+\cdots+x_{r-1} t_{r-1}+q_{r} t_{r} n_{r}+s_{r} t_{r} .
$$

Como $n_{r} t_{r} \in \mathbb{N} t_{0}+\mathbb{N} t_{1}+\cdots+\mathbb{N} t_{r-1}$, sigue que existe $\lambda_{0}, \lambda_{1}, \ldots, \lambda_{r-1}$, tal que

$$
n=\lambda_{0} t_{0}+\lambda_{1} t_{1}+\cdots+\lambda_{r-1} t_{r-1}+s_{r} t_{r}, \operatorname{con} 0 \leq s_{r}<n_{r} .
$$

Para $\lambda_{r-1}$ y $n_{r-1}$, existe $s_{r-1}$ y $q_{r-1}$, tal que

$$
\lambda_{r-1}=n_{r-1} q_{r-1}+s_{r-1} \quad \text { con } 0 \leq s_{r-1}<n_{r-1},
$$

en (18), se tiene

$$
n=\lambda_{0} t_{0}+\cdots+n_{r-1} q_{r-1} t_{r-1}+s_{r-1} t_{r-1}+s_{r} t_{r}
$$

con $0 \leq s_{r-1}<n_{r-1}$ y $0 \leq s_{r}<n_{r}$. Así de manera sucesiva obtenemos el resultado.

$2) \Rightarrow 3)$ Por el corolario 2.15 sigue que para $n \in A$, entonces $n=s_{0} t_{0}+s_{1} t_{1}+\cdots+s_{r} t_{r}$ con $0 \leq s_{i}<n_{i}, i, \ldots, r$ y $s_{0} \leq 0$, por tanto $s_{0}=0$. Consecuentemente

$$
A=\left\{\sum_{i=1}^{r} s_{i} t_{i}, \text { con } 0 \leq s_{i}<n_{i}, i=1, \text { ldots }, r\right\} .
$$

$3) \Rightarrow 4$ ) Por el lema 2.5 sigue

$$
f=a_{t_{0}-1}-t_{0}+1
$$

donde

$$
a_{t_{0}-1}=\operatorname{máx} A=\sum_{i=1}^{r}\left(n_{i}-1\right) t_{i} .
$$


$4) \Rightarrow 1$ Sea la sucesión derivada $t_{0}^{\prime}, t_{1}^{\prime}, \ldots, t_{r-1}^{\prime}$ de $t_{0}, t_{1}, \ldots, t_{r}$ y sea $H^{\prime}$ su semigrupo generado, esto es

$$
H^{\prime}=\sum_{i=0}^{r-1} \mathbb{N} t_{i}^{\prime}
$$

Entonces

$$
H^{\prime} n_{r} \subset H .
$$

Afirmación 1. El conductor $f^{\prime}$ de $H^{\prime}$ esta dado por

$$
f^{\prime}=\sum_{i=1}^{r-1}\left(n_{i}^{\prime}-1\right) t_{i}^{\prime}-t_{0}^{\prime}+1
$$

pues caso contrario por la Proposición 2.17 tenemos

$$
f^{\prime}<\sum_{i=1}^{r-1}\left(n_{1}^{\prime}-1\right) t_{i}^{\prime}-t_{0}^{\prime}+1
$$

sigue $f^{\prime} \leq \sum_{i=1}^{r-1}\left(n_{1}^{\prime}-1\right) t_{i}^{\prime}-t_{0}^{\prime} \in H^{\prime}$ y por 19

$$
f-1=\sum_{i=1}^{r}\left(n_{1}-1\right) t_{i}-t_{0} \in H
$$

que es un absurdo!. Por tanto la afirmación es verdadera.

Sigue por hipótesis inductiva que $t_{0}^{\prime}, t_{1}^{\prime}, \ldots, t_{r-1}^{\prime}$ es sucesión agradable. Luego $n_{i}^{\prime} t_{i}^{\prime} \in \sum_{j<i} \mathbb{N} t_{j}$, $i=1, \ldots, r-1$, entonces

$$
n_{i} t_{i} \in \sum_{j<i} \mathbb{N} t_{j}, \quad i=1, \ldots, r-1
$$

Afirmación 2. $H^{\prime}$ es simétrico. En efecto como $t_{0}^{\prime}, t_{1}^{\prime}, \ldots, t_{r-1}^{\prime}$ es agradable sigue del item 3. que

$$
a_{t_{0}^{\prime}-1}=\operatorname{máx} A^{\prime}=\sum_{i=1}^{r-1}\left(n_{1}^{\prime}-1\right) t_{i}^{\prime}-t_{0}^{\prime}
$$

luego $a_{t_{0}^{\prime}-1}-t_{0}^{\prime} \notin H^{\prime}$, sigue por la proposición 2.8 que $H^{\prime}$ es simétrico.

$t_{r} \in H^{\prime}$. En efecto si $t_{r} \notin H^{\prime}$, sigue por ser $H^{\prime}$ simétrico que para $z=f-1$, se tiene $f^{\prime}-1-t_{r} \in H^{\prime}$, sigue $n_{r}\left(f^{\prime}-1\right)-n_{r} t_{r} \in H^{\prime} n_{r}$, luego por (19)

$$
n_{r}\left(f^{\prime}-1\right) \in n_{r} t_{r}+n_{r} H \subset H
$$

Consecuentemente

$$
f-1=\left(n_{r}-1\right) t_{r}+\sum_{i=1}^{r-1}\left(n_{1}-1\right) t_{i}-t_{0}=\left(n_{r}-1\right) t_{r}+\left(f^{\prime}-1\right) n_{r} \in H,
$$

que es un absurdo!. Por tanto la afirmación es verdadera.

Afirmación 4. $n_{r} t_{r} \in \sum_{j=1}^{r-1} \mathbb{N} t_{j}$ En efecto como $t_{r} \in H^{\prime}$, sigue $t_{t} \in \sum_{j=1}^{r-1} \mathbb{N} t_{j}$, luego $n_{r} t_{r} \in \sum_{j=1}^{r-1} \mathbb{N} t_{j}$ y la afirmación es verdadera.

Por la afirmación 4. y (20) sigue que $t_{0}, t_{1}, \ldots, t_{r}$ sucesión agradable.

Corolario 2.22 Un subsemigrupo aditivo de $\mathbb{N}$ que es generado por una sucesión agradable, es simétrico. 
Demostración Sea $t_{0}, t_{1}, \ldots, t_{r}$ una sucesión agradable y $H=\sum_{i=1}^{r} \mathbb{N} t_{i}$, luego por el teorema 2.21 item 3 . sigue que

$$
a_{t_{0}-1}=\operatorname{máx} H=\sum_{i=1}^{r-1}\left(n_{i}-1\right) t_{i}-t_{0},
$$

luego $a_{t_{0}-1}-t_{0} \notin H$ consecuentemente por la por la proposición $2.8 H$ es simétrico.

La recíproca no es cierta pues si escojamos el semigrupo generado por 8, 10,11, 13.Entonces

$$
\mathbb{N} \backslash H=\{1,2,3,4,5,6,7,9,12,14,15,17,25\},
$$

luego por la Proposición $2.7 H$ es simétrico. Pero por definición la sucesión 8, 10,11,13 no es agradable.

\section{Conclusiones}

La teoría de semigrupos es un campo muy interesante de investigación ya que aporta resultados que posteriormente usaremos en el estudio de una singularidad de una curva algebroide plana.

El teorema de Angermuller es un resultado que asegura la existencia de conductor de un semigrupo que es un invariante que caracteriza al semigrupo. 


\section{REFERENCIAS BIBLIOGRÁFICAS}

[1] G. Angermüller. Die Wertehalbgruppe einer ebenen irreduziblen algebroiden Kurve. Math. Zeitschr. 153, 1977. p. 267-282.

[2] A. Hefez. Singularidades de curvas irredutiveis planas. The sixth workshop on real and complex singulariies. USP-Sao Carlos,p. 17-21 July ,2000. 